\title{
Évaluation en laboratoire de deux trousses de test au point de service pour le dépistage de la syphilis infectieuse
}

\begin{abstract}
Raymond SW Tsang ${ }^{1 *}$, Michelle Shuel ${ }^{1}$, Kristy Hayden ${ }^{1}$, Paul Van Caeseele ${ }^{2}$, Derek Stein ${ }^{2,3}$
\section{Résumé}

Contexte : La syphilis est une maladie sexuellement transmissible dont le tableau clinique peut être atypique. Les tests de laboratoire classiques pour confirmer le diagnostic ne sont pas assez rapides pour influencer la décision clinique sur le traitement et le dépistage des contacts. Des trousses de test rapide au point de service (TRPS) peuvent s'avérer utiles pour le contrôle des maladies infectieuses. Néanmoins, au Canada, il n'existe pas encore de TRPS pour la syphilis. L'objectif de la présente étude est d'évaluer deux TRPS (test RevealMD Rapid de détection d'anticorps dirigés contre le tréponème [Treponema pallidum] et test de dépistage et de confirmation de la syphilis DPP ${ }^{\circledR}$ pour la détection de la syphilis infectieuse.
\end{abstract}

Méthodes : Une centaine de prélèvements sériques ayant un statut sérologique de syphilis connu, d'après les résultats des tests tréponémique et non tréponémique, ont été analysés en laboratoire à l'aveugle, avec deux TRPS par deux opérateurs indépendants. Les résultats des TRPS ont été analysés dans le but d'évaluer la capacité de ces derniers à détecter la syphilis infectieuse.

Résultats : Le TRPS RevealMD Rapid TP a montré une sensibilité globale de $95,0 \%$ et une spécificité de $83,3 \%$, tandis que le TRPS DPP Syphilis Screen and Confirm Assay a montré une sensibilité de $87,5 \%$ et une spécificité de 98,3\%. Les deux TRPS ont donné une sensibilité de $100 \%$ sur les prélèvements de syphilis active avec des titres de plus de 1:4 sur le test du Venereal Disease Research Laboratory (VDRL), mais leur sensibilité a diminué pour les prélèvements avec des titres de VDRL faibles. Les deux TRPS ont donné des résultats faiblement ou très faiblement réactifs sur $11,3 \%$ à $25,0 \%$ des prélèvements positifs à la présence d'anticorps dirigés contre les antigènes du tréponème.

Conclusion : Cette évaluation en laboratoire a montré des résultats prometteurs pour la détection de la syphilis infectieuse par les deux TRPS. D'autres évaluations sur le terrain seront nécessaires pour confirmer ces résultats préliminaires.
Cette oeuvre est mise à la disposition selon les termes de la licence internationale Creative Commons Attribution 4.0

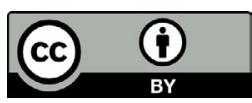

Affiliations

${ }^{1}$ Unité de diagnostic de la syphilis, Laboratoire national de microbiologie, Agence de la santé publique du Canada, Winnipeg, MB

${ }^{2}$ Laboratoire provincial Cadham, Winnipeg, MB

${ }^{3}$ Département de microbiologie médicale et des maladies infectieuses, Université du Manitoba, Winnipeg, MB

\author{
${ }^{\star}$ Correspondance : \\ raymond.tsang@phac-aspc.gc.ca
}

Citation proposée : Tsang RSW, Shuel M, Hayden K, Van Caeseele P, Stein DR. Évaluation en laboratoire de deux trousses de test au point de service pour le dépistage de la syphilis infectieuse. Relevé des maladies transmissibles au Canada 2022;48(2/3):93-9. https://doi.org/10.14745/ccdr.v48i23a05f

Mots-clés : syphilis infectieuse, tests au point de service

\section{Introduction}

La syphilis infectieuse est en hausse en Amérique du Nord depuis le début des années $2000(1,2)$. Ces dernières années, l'augmentation a été importante : au Canada, le taux de syphilis infectieuse s'est multiplié par 3,4 en 10 ans, passant d'un peu moins de cinq cas par 100000 habitants en 2009 à environ dix-sept cas par 100000 habitants en 2018 (3). De plus, on a observé un changement dans les taux croissants de syphilis infectieuse chez les femmes en âge de procréer (15 à 39 ans) aux États-Unis et au Canada $(2,4)$, ce qui a entraîné une augmentation de la syphilis congénitale dans les deux pays. Les raisons de l'augmentation du nombre de cas de syphilis au Canada ont récemment fait l'objet de discussions (5), et peuvent comprendre un meilleur accès aux tests, une sensibilité accrue des immunoessais enzymatiques utilisés dans le dépistage de 
la syphilis, une interruption des relations sexuelles protégées et des changements dans le comportement social associés à la disponibilité de plateformes de médias sociaux pour faciliter les rencontres sexuelles récréatives. Pour lutter contre cette hausse, il a également été suggéré de sensibiliser davantage le public à la syphilis, de recueillir des données de surveillance en temps opportun et de manière plus approfondie afin de contribuer à I'élaboration d'interventions qui ciblent les comportements à risque ou différents groupes ethniques à risque, et de meilleures méthodes d'analyse qui peuvent donner des résultats immédiats pour permettre un traitement et un dépistage plus précoces des contacts (6).

On nomme souvent la syphilis « la grande imitatrice » (7); le diagnostic clinique et en laboratoire de la syphilis infectieuse peut se révéler difficile. Le test de diagnostic et de confirmation le plus rapide pour la syphilis infectieuse est la microscopie en champ sombre, qui permet de rechercher les spirochètes mobiles dans les prélèvements cliniques, mais cette méthode est maintenant quasi inexistante au Canada en raison de l'expertise technique requise et de la relative insensibilité de cette méthode. De façon conventionnelle, la syphilis est diagnostiquée sérologiquement en mesurant les anticorps dirigés contre les antigènes du tréponème (e.g. en utilisant des immunoessais par microparticules chimiominescentes [CMIA] qui détectent les anticorps $\lg G$ et $\lg \mathrm{M}$ ) et les antigènes ne provenant pas du tréponème (e.g. en utilisant les tests rapides de la réagine plasmatique [RPR] ou les tests de la Venereal Disease Research Laboratory [VDRL]). Les patients atteints de syphilis active ou infectieuse affichent des résultats positifs avec le test tréponémique et le test non tréponémique, tandis que les patients ayant des antécédents de syphilis n'affichent des résultats positifs qu'avec le test tréponémique. Bien que ces tests sérologiques conventionnels et les nouvelles méthodes de diagnostic faisant intervenir les tests d'amplification en chaîne par polymérase soient exacts et sensibles, les résultats ne sont pas disponibles assez rapidement pour permettre des décisions cliniques sur le traitement des patients et le dépistage des contacts. De plus, il n'existe actuellement sur le marché aucun test d'amplification de l'acide nucléique autorisé pour le dépistage de la syphilis.

Bien que des tests de dépistage de la syphilis au point de service (TRPS) aient été élaborés au début des années 2000 et qu'ils aient été utilisés dans un certain nombre de pays à ressources limitées qui n'ont pas les infrastructures de laboratoire, l'équipement et le personnel qualifié nécessaires pour effectuer des tests de laboratoire conventionnels, leur utilisation dans les pays riches en ressources est moins bien définie. En effet, les US Centers for Disease Control and Prevention et l'American Association of Public Health Laboratories, en consultation avec d'autres experts en maladies sexuellement transmissibles, ont conclu en 2017 qu'il n'y avait pas suffisamment de données pour recommander l'utilisation des TRPS pour les tests systématiques de syphilis aux États-Unis (8).
Le 2 octobre 2020, la US Food and Drug Administration a délivré une licence pour deux trousses TRPS pour la syphilis (9) : le Syphilis Health Check POC assay (Diagnostic Direct LLC, Stone Harbor, New Jersey ou Trinity Biotech, Jamestown, New York) et le DPP POCT for HIV and Syphilis serology (Chembio Diagnostic System, Medford, New York). Cependant, certaines évaluations de ces trousses de test étaient fondées sur un petit nombre de prélèvements positifs ainsi que sur des prélèvements sériques obtenus par ponction veineuse plutôt que par prise de sang au bout du doigt, zone de prélèvement de choix pour le TRPS (en raison de la facilité d'obtention du spécimen). À partir de prélèvements sanguins pris sur le bout du doigt, le Syphilis Health Check POCT présentait une sensibilité de $100 \%$ (avec sept prélèvements positifs) comparativement aux tests de référence consensuels que sont le RPR et l'immunoessai enzymatique tréponémique. Cette sensibilité a baissé à 77,8 \% (avec 18 prélèvements positifs) par rapport au Syphilis Health Check POCT effectué avec du sérum en laboratoire, et à seulement $50,0 \%$ de sensibilité (avec 16 prélèvements positifs) par rapport à l'immunoessai enzymatique tréponémique (10). Des difficultés à lire les résultats du Syphilis Health Check POCT ont également été signalées. De fait, des prélèvements détectés positifs par ce dernier s'avèrent négatifs par consensus lors des tests de référence. Selon une étude de 2016 qui mentionne une sensibilité élevée $(94,7 \%)$ et une spécificité élevée (100\%) pour le DPP POCT for HIV and Syphilis serology, les résultats pour la composante syphilis du TRPS ont été fondés sur le dépistage de prélèvements sériques et la comparaison des données avec celles obtenues par le test d'agglutination de particules de Treponema pallidum (T. pallidum) (11). À I'heure actuelle, aucun TRPS pour la syphilis n'est autorisé au Canada.

La présente étude visait à évaluer deux TRPS pour la détection de la syphilis infectieuse (I'un avec des antigènes du tréponème, et l'autre avec des antigènes issus du tréponème et non issus du tréponème) et à rendre compte de leur performance préliminaire à partir d'analyses effectuées en laboratoire à l'aide de prélèvements sériques dont les résultats à des tests sérologiques de syphilis étaient connus.

\section{Matériel et méthodes}

\section{Prélèvements sériques de patients et tests par sérologie conventionnelle de la syphilis}

Une centaine de prélèvements sériques provenant de sujets individuels, identifiés comme étant soit positifs soit négatifs pour la syphilis au Laboratoire provincial Cadham (Winnipeg, Manitoba), ont été fournis à l'aveugle (en ce qui concerne les résultats des tests de syphilis) au Laboratoire national de microbiologie aux fins d'évaluation par TRPS. Les prélèvements ont été définis comme étant positifs à la syphilis si les tests effectués par CMIA étaient positifs pour les anticorps dirigés contre les antigènes du tréponème. Les prélèvements ont été définis comme étant négatifs à la syphilis lorsque les tests par 
CMIA donnaient des résultats négatifs. Un RPR qualitatif a été effectué sur les prélèvements positifs selon le CMIA, suivi d'un test VDRL quantitatif effectué au Laboratoire provincial Cadham. Le RPR et le VDRL sont tous deux considérés comme des tests non tréponémiques qui détectent des anticorps non tréponémiques, et les résultats positifs des tests RPR ou VDRL (et en présence de résultats positifs obtenus par (MIA) indiquent une syphilis active ou infectieuse. En outre, un autre test non tréponémique (VDRL) a été utilisé pour confirmer les résultats qualitatifs du RPR afin d'accroître la spécificité des données des tests non tréponémiques.

\section{Test au point de service}

Comme il n'y a pas encore de trousse de TRPS autorisée pour la vente au Canada, nous avons obtenu la permission du Programme d'accès spécial de Santé Canada afin de pouvoir nous procurer les tests utilisés dans le cadre de cette étude directement auprès des fournisseurs. En ce qui concerne lesdits tests, il s'agit du test rapide de dépistage et de confirmation de la syphilis DPP ${ }^{\circledR}\left(\right.$ DPP ${ }^{\circledR}$ Syphilis Screen and Confirm Assay) (ChemBio Diagnostic Systems, Inc., Medford, New York) et du test rapide RevealMD de détection d'anticorps dirigés contre le tréponème (RevealMD Rapid TP Antibody test) (MedMira Laboratories, Inc., Halifax, Nouvelle-Écosse). Le TRPS Reveal Rapid TP Antibody détecte des anticorps dirigés contre des peptides synthétiques qui ressemblent à des antigènes recombinants de T. pallidum et peut être effectué sur des prélèvements sériques, sanguins ou de plasma (12). Le TRPS DPP Syphilis Screen and Confirm permet de détecter des anticorps dirigés contre des antigènes du tréponème ainsi que contre ceux non issus du tréponème dans des prélèvements sériques, sanguins ou de plasma (13).

Deux techniciens de laboratoire qualifiés du Laboratoire national de microbiologie ont effectué des tests indépendants sur chacun des 100 prélèvements sériques avec les deux trousses de TRPS en suivant les instructions du fabricant. Les résultats ont été lus manuellement et enregistrés comme réactifs, faiblement réactifs ou très faiblement réactifs, même si tout changement d'intensité de la couleur dans la zone de test est considéré comme réactif selon la notice pour le TRPS Reveal Rapid TP Antibody et le TRPS DPP Syphilis Screen and Confirm. Nous avons choisi de noter les résultats des tests de façon plus détaillée, car la lecture de l'intensité du changement de couleur est subjective et les difficultés de lecture de certains TRPS de syphilis ont été décrites par d'autres chercheurs. Les résultats faiblement réactifs et très faiblement réactifs ont été déterminés par comparaison avec les réactions des prélèvements témoins ainsi qu'avec d'autres prélèvements réactifs obtenus au cours de la même période. Lorsque des résultats discordants étaient obtenus par les deux opérateurs indépendants, le test était fait une deuxième fois par les deux opérateurs et si les résultats étaient concordants lors du deuxième test, ces derniers étaient utilisés. En aucun cas, des résultats discordants importants $n$ 'ont été observés, par exemple la réaction chez un opérateur et la non-réaction chez l'autre.
Une fois que les 100 échantillons ont été testés par les deux opérateurs à l'aide des deux TRPS, les résultats ont été fournis aux experts cliniques du Laboratoire provincial Cadham, qui ont ensuite divulgué les résultats par CMIA, ainsi que les résultats du RPR et VDRL aux experts cliniques du Laboratoire national de microbiologie pour effectuer l'analyse. La sensibilité (pourcentage des prélèvements pour la syphilis infectieuse testés positifs), la spécificité (pourcentage des prélèvements sans syphilis infectieuse testés négatifs), le taux de faux positifs (pourcentage des prélèvements sans syphilis infectieuse testés positifs) et le taux de faux négatifs (pourcentage des prélèvements pour la syphilis infectieuse testés négatifs) ont été calculés pour les deux TRPS destinés à la détection de la syphilis infectieuse.

\section{Résultats}

\section{Caractéristiques des échantillons cliniques}

Sur les 100 échantillons fournis pour les évaluations des TRPS, 80 étaient réactifs ou positifs par CMIA (échantillon sur le seuil, $\mathrm{S} / \mathrm{CO}$, les valeurs variaient de 1,11 à 27,11 , moyenne $=15,68$, médiane $=16,81$ ) et 20 étaient non réactifs ou négatifs (les valeurs $\mathrm{S} / \mathrm{CO}$ variaient de 0,03 à 0,14 , moyenne $=0,61$, médiane $=0,05)$. Les 20 échantillons négatifs par CMIA n'ont pas fait l'objet d'autres tests et ont été considérés comme étant négatifs pour la syphilis tant pour les infections actives que pour les infections antérieures. Les 80 échantillons positifs par CMIA ont été divisés en deux : 40 échantillons qui n'étaient pas réactifs par RPR (ce qui suggère l'absence d'infections actives de syphilis, mais avec des infections antérieures de syphilis traitées) et 40 échantillons qui étaient réactifs par RPR (ce qui suggère des infections actives de syphilis). Parmi les 40 échantillons positifs par CMIA et réactifs par RPR, six n'étaient que faiblement réactifs par RPR. De ces six échantillons faiblement réactifs par $R P R$, cinq ont été testés par VDRL et des résultats réactifs ont été obtenus avec un échantillon non dilué. L'échantillon restant, positif par CMIA, et faiblement réactif au RPR, était réactif au VDRL au taux de dilution 1:2. Parmi les 34 échantillons réactifs par CMIA et par RPR, 14 avaient des titres de VDRL faibles ou nuls ( $n=7$ avec des titres de VDRL de 1:2, $n=6$ avec des titres de VDRL de 1:4, et $n=1$ était réactif lors du test VDRL non dilué), alors que 20 échantillons avaient des titres de VDRL d'au moins 1:8.

\section{Performance des tests Reveal Rapid Treponema pallidum Antibody et DPP Syphilis Screen and Confirm}

Le tableau 1 montre les résultats du TRPS Reveal Rapid TP Antibody et du TRPS DPP Syphilis Screen and Confirm sur les trois catégories de prélèvements sériques (aucune infection à la syphilis, infection antérieure et infection active). Des prélèvements sériques provenant de sujets sans antécédents de syphilis ni syphilis active étaient non réactifs aux deux TRPS. Seulement 10 des 40 échantillons classés dans la catégorie 
« syphilis antérieure » ont été réactifs au TRPS Reveal Rapid TP Antibody. Le TRPS DPP Syphilis Screen and Confirm a détecté des anticorps dirigés contre les antigènes du tréponème dans 19 des 40 échantillons prélevés chez des sujets ayant des antécédents de syphilis; un seul échantillon était réactif pour les antigènes du tréponème et pour ceux non issus du tréponème, tandis que les 20 autres étaient non réactifs pour les deux types $d^{\prime}$ antigènes.

Le pourcentage d'échantillons provenant des sujets atteints de syphilis active et réactifs aux deux TRPS (Reveal Rapid TP Antibody et DPP Syphilis Screen and Confirm) est corrélé aux résultats quantitatifs du test non tréponémique, et cette corrélation est particulièrement évidente avec le DPP Syphilis Screen and Confirm (voir tableau 1).

Bien que les résultats très faiblement réactifs ont été jugés, avec une grande prudence, non réactifs (indiqués entre parenthèses dans le tableau 1 et dans le tableau 2), moins de prélèvements provenant de sujets ayant des antécédents de syphilis ou souffrant de syphilis active ont été jugés réactifs pour les deux TRPS. Vingt prélèvements ont été testés en utilisant le Reveal Rapid TP Antibody, et ce test a donné une réaction faible ( $n=12$; soit trois prélèvements de syphilis antérieure et neuf de syphilis active) ou très faiblement réactifs ( $n=8$; soit six prélèvements de syphilis antérieure et deux de syphilis active). Dans le cas du DPP Syphilis Screen and Confirm, douze échantillons ont donné une faible réactivité ( $n=1$; syphilis antérieure) ou une très faible réactivité $(n=11$; soit six prélèvements de syphilis antérieure et cinq de syphilis active) de la présence d'antigènes du tréponème, tandis que neuf échantillons ( $n=8$ prélèvements de syphilis active et un de syphilis antérieure) ont donné des résultats très faiblement réactifs de la présence d'antigènes du tréponème (données non présentées).

Le tableau 2 compare les caractéristiques de performance des deux TRPS de syphilis pour les 100 prélèvements sériques qui ont été répartis dans les catégories suivantes : syphilis non traitée, syphilis antérieure guérie, syphilis active ou syphilis infectieuse. La sensibilité des deux TRPS pour la détection de la syphilis infectieuse semble être affectée par les résultats quantitatifs des tests non tréponémiques effectués sur les échantillons. Les deux TRPS ont donné une sensibilité de $100 \%$ sur les échantillons avec des titres VDRL supérieurs à 1:4, mais la sensibilité a diminué progressivement pour les échantillons avec des titres VDRL inférieurs (tableau 2), et les résultats du DPP Syphilis Screen and Confirm semblent être plus affectés encore lorsqu'il s'agit d'échantillons avec des titres VDRL de 1:2 à 1:4 ou moins. Bien que le Reveal Rapid TP Antibody ait une sensibilité plus élevée pour la détection de la syphilis active, le DPP Syphilis Screen and Confirm a une plus grande spécificité.

\section{Tableau 1 : Résultats de deux trousses de test rapide au point de service montrant le nombre d'échantillons réactifs et le nombre d'échantillons non réactifs sur 100 prélèvements sériques ayant des résultats sérologiques de syphilis conventionnels connus}

\begin{tabular}{|c|c|c|c|c|c|c|c|c|c|c|c|c|c|}
\hline \multirow{2}{*}{$\begin{array}{l}\text { Statut en } \\
\text { matière de } \\
\text { syphilis }^{\text {a }}\end{array}$} & \multirow{2}{*}{$\begin{array}{l}\text { Nombre } \\
\text { de } \\
\text { spécimens }\end{array}$} & \multicolumn{4}{|c|}{$\begin{array}{l}\text { TRPS Reveal Rapid } \\
\text { T. pallidum Antibody }\end{array}$} & \multicolumn{8}{|c|}{$\begin{array}{l}\text { TRPS DPP Syphilis Screen and Confirmc } \\
\text { présenté comme réactions aux antigènes du tréponème/non issus du } \\
\text { tréponème }\end{array}$} \\
\hline & & $\mathbf{R}^{\mathbf{d}}$ & $(R)^{d}$ & $N^{e}$ & $(N R)^{e}$ & $R / R^{d}$ & $(R / R)^{d}$ & $R^{d} / N R$ & $(R / N R)^{d, e}$ & $N R^{e} / R^{d}$ & $(N R / R)^{d, e}$ & $N R^{e} / N^{e}{ }^{e}$ & $(\mathrm{NR} / \mathrm{NR})^{\mathrm{e}}$ \\
\hline $\begin{array}{l}\text { Aucune } \\
\text { syphilis }\end{array}$ & 20 & 0 & 0 & 20 & 20 & 0 & 0 & 0 & 0 & 0 & 0 & 20 & 20 \\
\hline $\begin{array}{l}\text { Syphilis } \\
\text { antérieure }\end{array}$ & 40 & 10 & 4 & 30 & 36 & 1 & 0 & 18 & 13 & 0 & 0 & $20^{f}$ & $26^{f}$ \\
\hline $\begin{array}{l}\text { Syphilis } \\
\text { active }^{9}\end{array}$ & 40 & 38 & 36 & 2 & 4 & 35 & 27 & 5 & 8 & 0 & 0 & 0 & 5 \\
\hline $\begin{array}{l}\text { Avec titrage } \\
\text { VDRL } \\
\text { supérieur à } \\
1: 4\end{array}$ & 20 & 20 & 20 & 0 & 0 & 20 & 19 & 0 & 0 & 0 & 0 & 0 & 1 \\
\hline $\begin{array}{l}\text { Avec titrage } \\
\text { VDRL } \\
1: 2 \text { à } 1: 4\end{array}$ & 13 & 12 & 11 & 1 & 2 & 10 & 7 & 3 & 3 & 0 & 0 & 0 & 3 \\
\hline $\begin{array}{l}\text { VDRL non } \\
\text { dilué et } \\
\text { RPR FR }^{\mathrm{h}}\end{array}$ & 7 & 6 & 5 & 1 & 2 & 5 & 1 & 2 & 5 & 0 & 0 & 0 & 1 \\
\hline
\end{tabular}

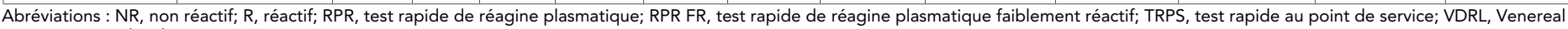
Disease Research Laboratory

${ }^{a}$ Aucune syphilis, immunoessai par microparticules chimiominescentes (CMIA) négatif; syphilis antérieure, CMIA positif, mais RPR non réactif; syphilis active, CMIA positif et RPR ou VDRL réactif

b MedMira Laboratories, Inc., Halifax, Nouvelle-Écosse

c ChemBio Diagnostic Systems, Inc., Medford, New York. Les résultats du TRPS DPP Syphilis Screen and Confirm ont été présentés comme réactions aux antigènes du tréponème/réactions aux antigènes non issus du tréponème

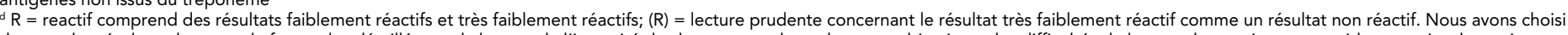

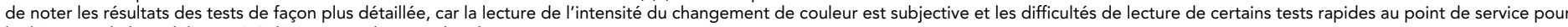
la détection de la syphilis ont été décrites par d'autres chercheurs

e $N R=$ non réactif; $(N R)=$ lecture prudente et résultats très faiblement réactifs inclus

${ }^{\dagger}$ Prélèvement non inclus si le résultat au TRPS DPP Syphilis Screen and Confirm était non concluant

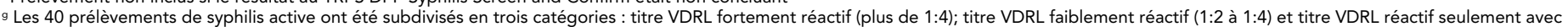
prélèvement sérique non dilué ou RPR qualitatif faiblement réactif

h Un échantillon faiblement réactif au RPR et un titrage VDRL 1:2; un échantillon réactif au RPR et réactif VDRL non dilué; et cinq échantillons faiblement réactifs au RPR et réactif VDRL non dilué

Page 96 RMTC • février/mars 2022 • volume 48 numéro 2/3 
Table 2 : Performance du test rapide au point de service Reveal Rapid TP Antibody et du test rapide au point de service DPP Syphilis Screen and Confirm pour la détection de la syphilis active ou infectieuse à partir d'une évaluation en laboratoire de 100 prélèvements sériques définis par des tests sérologiques de syphilis conventionnels ${ }^{\mathrm{a}}$

\begin{tabular}{|l|r|r|r|r|}
$\begin{array}{c}\text { Caractéristiques } \\
\text { de la performance } \\
\text { du test rapide de } \\
\text { syphilis au point de } \\
\text { service }\end{array}$ & $\begin{array}{r}|c| \\
\text { TRPS } \\
\text { Rapid T. pallidum } \\
\text { Antibody }\end{array}$ & $\begin{array}{r}\text { Revel } \\
\text { TRPSc DPP } \\
\text { Syphilis Screen } \\
\text { and Confirm }\end{array}$ \\
\hline Sensibilité générale & $95,0 \%$ & $(90,0 \%)$ & $87,5 \%$ & $(67,5 \%)$ \\
\hline Sensibilitéf & $100 \%$ & $(100 \%)$ & $100 \%$ & $(95,0 \%)$ \\
\hline Sensibilitég & $92,3 \%$ & $(84,6 \%)$ & $76,9 \%$ & $(53,8 \%)$ \\
\hline Sensibilitéh & $85,7 \%$ & $(71,4 \%)$ & $71,4 \%$ & $(14,3 \%)$ \\
\hline Spécificité & $83,3 \%$ & $(93,3 \%)$ & $98,3 \%$ & $(100 \%)$ \\
\hline Taux de faux positifs & $16,7 \%$ & $(6,7 \%)$ & $1,7 \%$ & $(0 \%)$ \\
\hline Taux de faux négatifs & $5,0 \%$ & $(10,0 \%)$ & $12,5 \%$ & $(32,5 \%)$ \\
\hline
\end{tabular}

Abréviation : TRPS, test rapide au point de service

Les tests sérologiques conventionnels de syphilis comprenaient un immunoessai par microparticules chimiominescentes (CMIA) suivi d'un test rapide de la réagine plasmatique (RPR) et/ou un test VDRL (Venereal Disease Research Laboratory) pour les prélèvements positifs au CMIA

MedMira Laboratories, Inc., Halifax, Nouvelle-Écosse

Les résultats faiblement réactifs et très faiblement réactifs ont été jugés réactifs (les résultats ont été lus de façon prudente, ce faisant les resultats très faiblement reactifs ont été jugés non réactifs). Nous avons choisi de noter les résultats des tests de façon plus détaillée, car la lecture de l'intensité du changement de couleur est subjective et les difficultés de lecture de certains chercheurs

ChemBio Diagnostic Systems, Inc., Medford, New York

La sensibilité globale était basée sur 40 prélèvements testés positifs au CMIA et réactifs ou faiblement réactifs au RPR. Les 40 prélèvements de syphilis active ont été subdivisés en trois catégories : titrage VDRL fortement réactif (plus de 1:4); titrage VDRL faiblement réactif (1:2 à 1:4) et titrage VDRL réactif seulement avec prélèvement sérique non dilué ou RPR qualitatif faiblement réactif

f Sur la base de 20 échantillons testés positifs au CMIA, réactifs au RPR et avec des titres supérieurs à 1:4 au VDRL

Sur la base de 13 échantillons testés positifs au CMIA, réactifs au RPR et avec des titres compris entre $1: 2$ et $1: 4$ au VDRL

${ }^{h}$ Sur la base de $n=7$ prélèvements testés positifs au CMIA, soit faiblement réactifs au RPR et/ou réactifs, non dilué, au VDRL

\section{Reproductibilité des résultats des tests au point de service menés par deux opérateurs indépendants}

Pour le Reveal Rapid TP Antibody, $91 \%$ des résultats des deux opérateurs étaient concordants tandis que pour le DPP Syphilis Screen and Confirm Assay, $94 \%$ des résultats de la composante tréponémique et $95 \%$ des résultats de la composante non tréponémique du test étaient concordants entre les deux opérateurs.

Des écarts mineurs sur neuf prélèvements ont été notés entre les deux opérateurs lors de la mise à l'essai du test Reveal Rapid TP Antibody, l'opérateur $n^{\circ} 1$ a indiqué neuf échantillons comme faiblement réactifs et l'opérateur $n^{\circ} 2$ en a signalé sept comme réactifs et deux comme très faiblement réactifs. Dans le cas du DPP Syphilis Screen and Confirm Assay, cinq résultats se sont avérés divergents pour la composante non tréponémique du test, l'opérateur $n^{\circ} 1$ a indiqué des résultats réactifs et l'opérateur $n^{\circ} 2$ a signalé des résultats très faiblement réactifs. En ce qui concerne la composante tréponémique du DPP Syphilis Screen and Confirm Assay, six résultats se sont avérés divergents, l'opérateur $n^{\circ} 1$ a indiqué des résultats réactifs et l'opérateur $n^{\circ} 2$ a signalé des résultats très faiblement réactifs.

\section{Discussion}

Cette étude de laboratoire a révélé que le Reveal Rapid TP Antibody et le DPP Syphilis Screen and Confirm avaient tous deux une sensibilité et une spécificité globales de $85,0 \%$ ou plus; le TRPS Reveal Rapid TP Antibody ayant une sensibilité globale plus élevée $(95,0 \%)$ et le TRPS DPP Syphilis Screen and Confirm ayant une meilleure spécificité (98,3\%) pour la détection de la syphilis infectieuse. Des prélèvements sériques provenant de sujets sans antécédents de syphilis ni syphilis active ont donné des résultats non réactifs très clairs aux deux TRPS. Étant donné que les prélèvements testés n'étaient pas des échantillons aléatoires prélevés sur la population, nous n'avons pas tenté d'extrapoler les résultats actuels pour calculer les valeurs prédictives positives et négatives de ces deux TRPS.

La plupart des trousses de TRPS pour la syphilis disponibles sur le marché utilisent des antigènes du tréponème pour détecter la présence $d$ 'anticorps dirigés contre ces antigènes et seulement une poignée de trousses s'appuient sur des antigènes du tréponème et sur ceux non issus du tréponème qui peuvent permettre de détecter la présence aussi bien d'anticorps dirigés contre les antigènes du tréponème que la présence $d$ 'anticorps non dirigés contre les antigènes du tréponème. Seuls les tests qui détectent les anticorps anti-tréponémiques peuvent permettre de différencier les patients atteints d'une infection active de ceux ayant eu une infection par le passé, car les anticorps anti-tréponémiques ont tendance à persister longtemps dans l'organisme après la disparition de l'infection à la suite d'un traitement réussi. La présence d'anticorps dirigés contre les antigènes du tréponème et $d$ 'anticorps dirigés contre les antigènes non issus du tréponème révèle une syphilis active et infectieuse, car les anticorps dirigés contre des antigènes non issus du tréponème ont généralement tendance à baisser graduellement et à disparaître le cas échéant quelques mois après que l'infection active soit guérie par un traitement.

Bien que le TRPS Reveal Rapid TP Antibody s'appuie sur des antigènes qui représentent les protéines du T. pallidum, et que le TRPS DPP Syphilis Screen and Confirm comporte également une composante qui s'appuie sur les antigènes du tréponème, les résultats suggèrent que les deux TRPS favorisent la détection de la syphilis infectieuse plutôt que de la syphilis antérieure traitée. La présence d'anticorps dirigés contre les antigènes du tréponème a été détectée dans seulement $25,0 \%$ (Reveal Rapid TP Antibody) et 48,7 \% (DPP Syphilis Screen and Confirm) des prélèvements sériques effectués sur des sujets ayant des antécédents de syphilis. Dans notre étude, par rapport aux résultats obtenus par CMIA, la sensibilité de la composante tréponémique du DPP Syphilis Screen and Confirm pour détecter 
la présence des anticorps anti-tréponémiques était de $100 \%$ pour les prélèvements sériques de syphilis active, mais n'était que de $48,7 \%$ pour les prélèvements sériques de syphilis antérieure. La sensibilité du Reveal Rapid TP Antibody à détecter la présence d'anticorps anti-tréponémiques était de 95,0\% pour les prélèvements de syphilis active, mais n'était que de $25,0 \%$ pour ceux de syphilis antérieure.

Cette étude montre que les deux TRPS évalués ont une bonne sensibilité et une bonne spécificité pour la détection de la syphilis infectieuse. Cependant, chez les patients ayant de faibles titres d'anticorps anti-tréponémiques, la performance des tests peut être compromise, ce qui confirme les résultats obtenus par d'autres chercheurs pour cette sous-catégorie des patients atteints de syphilis active $(10,14,15)$. Parmi les deux TRPS évalués, le Reveal Rapid TP Antibody aurait tendance à être moins affecté par de faibles titres d'anticorps anti-tréponémiques et les résultats obtenus avec ce test révèlent le maintien d'une sensibilité raisonnable de $92,3 \%$ à $85,7 \%$ (pour détecter la syphilis infectieuse) même pour des échantillons avec des titres d'anticorps VDRL de 1:2 à 1:4 ou réactifs seulement lorsque non dilués.

Avec le Reveal Rapid TP Antibody, 25,0 \% des prélèvements positifs au CMIA ont donné des résultats faiblement ou très faiblement réactifs. Avec le DPP Syphilis Screen and Confirm, nous avons constaté que 11,3\% (composante non tréponémique) et 15,0 \% (composante tréponémique) des échantillons positifs au CMIA ont donné des résultats faiblement ou très faiblement réactifs. Les résultats faiblement réactifs et surtout très faiblement réactifs peuvent donner lieu à des difficultés de lecture des résultats de ces tests sur le terrain par des opérateurs non formés. De plus, l'utilisation de prélèvements sanguins pris sur le bout du doigt peut rendre la lecture des résultats encore plus difficile. Une formation et des lignes directrices claires sur la façon de lire les résultats des TRPS pourraient être nécessaires. Certains TRPS sont munis d'un lecteur électronique (11), ce qui peut éviter des incohérences dans la lecture des résultats entre les échantillons et les opérateurs. Un autre défi potentiel dans la mise en œuvre des TRPS dans les cliniques dédiées aux infections sexuellement transmissibles ou dans les zones rurales provient du dépistage décentralisé de la syphilis et des difficultés qui en résultent dans la saisie des résultats des tests par la santé publique à des fins de surveillance et pour l'élaboration subséquente de politiques d'intervention en santé publique.

L'une des raisons évoquées pour expliquer l'augmentation récente de la syphilis infectieuse au Canada (dans les régions urbaines et éloignées ou rurales) est l'iniquité de l'accessibilité au sein du système de soins de santé, y compris les installations de dépistage des infections $(5,6)$. Il peut être difficile pour les populations vulnérables, qu'elles vivent en milieu urbain ou rural, de se rendre à ces installations en raison de logements précaires ou instables, de la méfiance envers le système de santé ou du manque d'installations de tests accessibles dans les localités éloignées. D'autres méthodes de test, comme l'utilisation de TRPS, peuvent contribuer à répondre au besoin créé par les situations de ces populations à risque. Le TRPS actuel ne fournit que des résultats qualitatifs de réactions positives ou négatives, et l'absence de résultats quantitatifs sur les titres d'anticorps peut empêcher leur utilisation pour surveiller la réponse au traitement et différencier les infections répétées ou les réinfections des infections antérieures. Cette méthode est en contraste avec la pratique actuelle qui consiste à utiliser les résultats quantitatifs d'un test non tréponémique (comme les titres RPR ou VDRL) pour surveiller soit une diminution des titres d'anticorps comme preuve d'une réponse positive au traitement, soit une augmentation du titrage d'anticorps en cas de nonréponse au traitement ou de réinfection.

\section{Limites}

L'interprétation des résultats de la présente étude comporte plusieurs limites. Premièrement, les prélèvements sériques recueillis pour la sérologie conventionnelle de la syphilis ont été utilisés au lieu d'échantillons sanguins pris sur le bout du doigt, qui est probablement la zone de prélèvement de choix lorsque ces tests seront utilisés sur le terrain. Deuxièmement, ces tests ont été effectués dans un environnement de laboratoire contrôlé par un personnel de laboratoire formé ayant de l'expérience dans la réalisation de tests de diagnostic clinique; par conséquent, la performance réelle de ces TRPS sur le terrain ou dans une situation réelle peut différer. Troisièmement, le petit nombre d'échantillons testés constitue une autre limite, en particulier dans la sous-catégorie des cas de syphilis active avec des titres de VDRL différents et, par conséquent, avec des données de sensibilité potentiellement inexactes. Enfin, I'utilité ou la performance d'un TRPS pourrait dépendre également du milieu ou de la prévalence de la maladie où l'utilisation du TRPS sera déployée.

\section{Conclusion}

Les deux TRPS évalués dans cette étude de laboratoire semblent présenter des résultats prometteurs pour la détection de la syphilis infectieuse, en particulier chez ceux dont les titres d'anticorps qui ne sont pas dirigés vers les antigènes du tréponème sont égaux ou supérieurs à 1:4, mais pas pour la détection de la syphilis antérieure. D'autres évaluations sur le terrain seront nécessaires pour confirmer les résultats de cette étude préliminaire. Des évaluations sur le terrain et des études cliniques offriront une plus grande expérience de l'utilisation des TRPS pour détecter la syphilis, ce qui pourrait éventuellement contribuer à un meilleur contrôle de la syphilis infectieuse et congénitale. Les données de ce type d'étude peuvent également être utiles pour l'autorisation future éventuelle de telles trousses de test au Canada. 


\section{Déclaration des auteurs}

R. S. W. T. - Conception de l'étude, analyse des résultats, préparation de la première ébauche

D. S. - Conception de l'étude

M. S. - Déroulement du test et analyse des résultats

K. H. - Déroulement du test et analyse des résultats

Tous les auteurs ont commenté et approuvé le manuscrit avant sa soumission.

Les résultats et l'opinion énoncés dans le présent manuscrit n'engagent que les auteurs et ne correspondent pas nécessairement à la position du Laboratoire national de microbiologie ni celle de l'Agence de la santé publique du Canada.

\section{Intérêts concurrents}

Aucun.

\section{Remerciements}

Les auteurs remercient M. L. Lam pour son aide dans la préparation de ce manuscrit et $A$. Gretchen pour la préparation des tests sérologiques.

\section{Financement}

Cette étude n'a reçu aucun financement externe et a été financée uniquement par l'Agence de la santé publique du Canada.

\section{Références}

1. Choudhri Y, Miller J, Sandhu J, Leon A, Aho J. La syphilis infectieuse et la syphilis congénitale au Canada, de 2010 à 2015. Relevé des maladies transmissibles au Canada. 2018;44(2):47-53. DOI

2. Centers for Disease Control and Prevention. National Overview Sexually Transmitted Disease Surveillance, 2019. Atlanta (GA): CDC; (modifié 2021; accédé 2021-06-20). https://www.cdc.gov/ std/statistics/2019/overview.htm\#syphilis

3. Agence de la santé publique du Canada. Syphilis infectieuse au Canada, 2018 (Infographique). Relevé des maladies transmissibles au Canada 2019;45(11):332. https://www.canada. $\mathrm{ca} / \mathrm{fr} /$ sante-publique/services/rapports-publications/relevemaladies-transmissibles-canada-rmtc/numero-mensuel/2019-45/ numero-11-7-novembre-2019/article-5-rapport-syphilisinfectieuse-canada-2009-2018.html
4. Agence de la santé publique du Canada. La syphilis infectieuse au Canada, 2019 (Infographique). Relevé des maladies transmissibles au Canada 2020;46(10):411. https://www.canada. $\mathrm{ca} / \mathrm{en} /$ public-health/services/reports-publications/canadacommunicable-disease-report-ccdr/monthly-issue/2020-46/ issue-10-october-1-2020/infectious-syphilis-2019.html

5. Singh AE. The return of syphilis in Canada: a failed plan to eliminate this infection. JAMMI 2019;4(4):215-7. DOI

6. Singh $A E$. How to address the resurgence of syphilis in Canada. CMAJ 2019;191(50):E1367-8. DOI

7. Klausner JD. The great imitator revealed: syphilis. Top Antivir Med 2019;27(2):71-4. PubMed

8. Association of Public Health Laboratories. Consultation on Laboratory Diagnosis of Syphilis. Meeting Summary Report. Silver Spring (MD): APHL; 2018. https://www.aphl.org/ aboutAPHL/publications/Documents/ID-2018Aug-SyphilisMeeting-Report.pdf

9. Gaydos CA, Manabe YC, Melendez JH. A narrative review of where we are with point-of-care sexually transmitted infection testing in the United States. Sex Transm Dis 2021;48 8S:S71-7. DOl PubMed

10. Fakile YF, Brinson M, Mobley V, Park IU, Gaynor AM. Performance of the Syphilis Health Check in clinic and laboratory-based settings. Sex Transm Dis 2019;46(4):250-3. DOI PubMed

11. Leon SR, Ramos LB, Vargas SK, Kojima N, Perez DG, Caceres CF, Klausner JD. Laboratory evaluation of a dual-path platform assay for rapid point-of-care HIV and syphilis testing. J Clin Microbiol 2016;54(2):492-4. DOI PubMed

12. MedMira Laboratories Inc. Reveal ${ }^{\mathrm{TM}}$ Rapid TP antibody test, package insert, Halifax (NS): MedMira; 2017. https://medmira. com/wp-content/uploads/2020/01/RSNYZIS0001EN-Reveal-TPPackage-Insert-new-format-eversion.pdf

13. Chembio Diagnostic Systems. DPP Screen and Confirm, package insert. Medford (NY); 2019. (package insert is only available by request). https://chembio.com/products/dppsyphilis-screen-confirm-europe/

14. Gliddon HD, Peeling RW, Kamb ML, Toskin I, Wi TE, Taylor MM. A systematic review and meta-analysis of studies evaluating the performance and operational characteristics of dual point-of-care tests for HIV and syphilis. Sex Transm Infect 2017;93(S4 Suppl 4):S3-15. DOI PubMed

15. Zorzi A, Cordioli M, Gios L, Del Bravo P, Toskin I, Peeling RW, Blondeel K, Cornaglia G, Kiarie J, Ballard R, Mirandola M. Field evaluation of two point-of-care tests for syphilis among men who have sex with men, Verona, Italy. Sex Transm Infect 2017;93(S4 Suppl 4):S51-8. DOI PubMed 